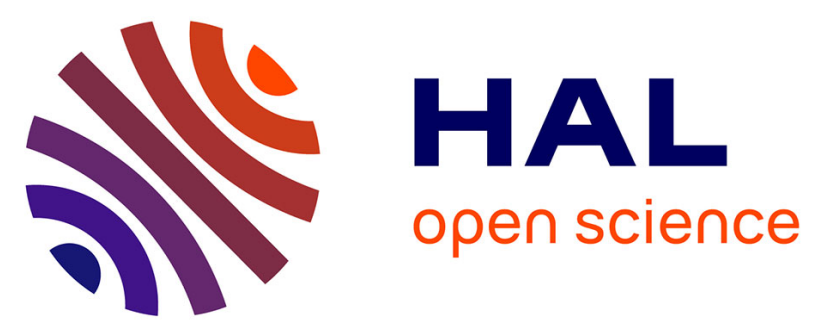

\title{
Optimisation aéro-structurale de la voilure d'un avion d'affaires par un jeu de Nash et un partage adapté des variables
}

Badr Abou El Majd, Jean-Antoine Désidéri, Abderrahmane Habbal

\section{- To cite this version:}

Badr Abou El Majd, Jean-Antoine Désidéri, Abderrahmane Habbal. Optimisation aéro-structurale de la voilure d'un avion d'affaires par un jeu de Nash et un partage adapté des variables. 9e Colloque national en calcul des structures, CSMA, May 2009, Giens, France. hal-01408408

\section{HAL Id: hal-01408408 \\ https://hal.science/hal-01408408}

Submitted on 4 Dec 2016

HAL is a multi-disciplinary open access archive for the deposit and dissemination of scientific research documents, whether they are published or not. The documents may come from teaching and research institutions in France or abroad, or from public or private research centers.
L'archive ouverte pluridisciplinaire HAL, est destinée au dépôt et à la diffusion de documents scientifiques de niveau recherche, publiés ou non, émanant des établissements d'enseignement et de recherche français ou étrangers, des laboratoires publics ou privés.

\section{(c)(1)}

Distributed under a Creative Commons Attribution| 4.0 International License 


\title{
Optimisation aéro-structurale de la voilure d'un avion d'affaires par un jeu de Nash et un partage adapté des variables
}

\author{
B. Abou El Majd ${ }^{1}$, J.-A. Désidéri ${ }^{2}$, A. Habbal ${ }^{3}$ \\ ${ }^{1}$ Université d'Avignon et des Pays du Vaucluse, Département de Mathématiques, \\ 33 rue Louis Pasteur, 84000 Avignon (badr.abou-el-majd@univ-avignon.fr) \\ ${ }^{2}$ INRIA Equipe-Projet OPALE, Centre de Sophia Antipolis Méditerranée, \\ 2004 Route des Lucioles, BP 93, 06902 Sophia Antipolis cedex (desideri@ sophia.inria.fr) \\ ${ }^{3}$ Université de Nice Sophia Antipolis, Laboratoire J.-A. Dieudonné, \\ Parc Valrose, 06108 Nice cedex 2 (habbal@ polytech.unice.fr)
}

\begin{abstract}
Résumé - On s'intéresse au problème de l'optimisation multidisciplinaire, lorsque les disciplines sont prises en compte par des critères qui sont des fonctionnelles de solutions distribuées d'équations aux dérivées partielles. Pour le cas de deux disciplines, on propose une stratégie dans laquelle l'optimisation est décomposée en deux phases : (a) une phase d'optimisation coopérative au cours de laquelle les critères sont améliorés à chaque itération, et (b) une phase d'optimisation concurrentielle réalisée par un jeu de Nash associé à un partage adapté des variables.
\end{abstract}

Mots clés - Ingénierie concourante, gradients, hessiens, systèmes propres, jeu de Nash.

\section{Introduction}

L'optimisation numérique de forme de la voilure d'un avion civil ou militaire en aérodynamique compressible soulève de nombreuses questions méthodologiques d'optimisation complexe car le problème est à la fois :

- multi-objectif, car on vise généralement à extrêmaliser plusieurs critères liés aux coefficients aérodynamiques, généralement sous contraintes ;

- multi-point, car ces critères sont critiques dans des phases différentes de l'enveloppe de vol; par exemple, la portance est critique en phase de décollage, d'atterrissage ou de manœuvre de vol subsonique, alors que réduire la traînée, qui conditionne la consommation en kérosène ou de manière équivalente le rayon d'action, est essentiel en régime transsonique ou supersonique de croisière ;

- multi-disciplinaire, car d'autres paramètres régis par des disciplines physiques autres que l'aérodynamique du vol sont importants : la répartition des charges structurales et thermiques, les émissions sonores et chimiques des moteurs, la signature radar (pour un avion furtif), les contraintes de mécanique du vol, etc,

sans même mentionner les contraintes de fabrication. Ces différentes disciplines physiques sont régies, dans les modèles de plus "grande fidélité", par des systèmes distribués d'équations aux dérivés partielles (EDP), tels que les équations d'Euler ou de Navier-Stokes pour l'aérodynamique, les équations de l'élasticité, de la thermique, de l'aéro-acoustique, ou de l'électromagnétisme (équations de Maxwell), etc. Dans ces différents domaines, les progrès des techniques numériques, 
notamment par volumes ou éléments finis, permettent aujourd'hui des simulations couplées de ces EDP. Les critères à optimiser sont alors des fonctionnelles des champs qui en résultent, et leur évaluation précise est possible, mais généralement assez coûteuse. Pour ces raisons, concevoir des méthodes aussi générales, robustes et économiques que possible pour optimiser concuremment plusieurs critères issus de physiques différentes est un défi aux experts du calcul, dont certains qualifient cette problématique d"'ingénierie concourante".

Dans notre équipe, nous réalisons des optimisations aérodynamiques de voilure par des techniques d'optimisation de forme paramétrique. On note $Y \in \mathbb{R}^{N}$ le vecteur de conception. Typiquement, on utilise l'approche par Free-Form Deformation [1] dans laquelle une géométrie initiale, ici donnée par un maillage tridimensionnel non structuré de volumes finis, est déformée par l'optimisation au moyen d'une transformation ponctuelle (3D) ayant pour support une "boîte" contenant la forme (2D) à optimiser, c'est-à-dire ici la surface de la voilure. Par exemple, on utilise fréquemment une déformation dans la base des produits tensoriels de trois polynômes de Bernstein, chacun associé à une direction de coordonnées, et de degré donné, ce qui généralise la représentation de Bézier. Les $N$ composantes du vecteur $Y$, nos variables de conception, sont alors des coordonnées ajustables de points de contrôle. Noter qu'on optimise la forme indirectement par le biais de la représentation symbolique posée a priori de sa déformation volumique, complètement indépendamment de la CAO qu'on a utilisé préalablement pour construire le maillage initial. En procédant de la sorte, on court-circuite le problème de la reconstruction d'un maillage admissible dont une frontière a été déformée, puisque nos variables servent à déplacer globalement tous les nœuds du maillage volumique. On pourra trouver des illustrations de cette approche dans [2] ainsi que des algorithmes multiniveaux inspirés des techniques multigrilles.

Ce cadre étant fixé, examinons maintenant comment le problème multi-objectif peut être traité. Une approche classique consiste à identifier numériquement la surface des points Pareto-optimaux. On rappelle qu'il s'agit des points de l'espace admissible dominés par aucun autre. Pour identifier ce front, Srinivas et Deb [3] ont proposé un algorithme très efficace : NSGA (Non-dominated sorting genetic algorithm). Il s'agit d'un algorithme génétique dans lequel on classe la population par fronts successifs de solutions non-dominées, et on définit la fonction d'adaptation au moyen de l'indice de front. Noter que Goldberg [4] a apporté un raffinement à l'algorithme par une technique de niching qui permet d'éviter l'accumulation de points proches sur un même front. Cet algorithme, désormais classique, est très puissant; par expérience on sait qu'il s'applique à des cas de front non convexe et même non continu. Mais l'algorithme est très coûteux car le front optimal est identifié comme l'enveloppe de toute une population que l'on fait évoluer par de nombreuses générations.

Comme alternative à l'identification du front des solutions Pareto-optimales, on explore la possibilité d'atteindre économiquement des solutions de compromis entre disciplines en réalisant des équilibres de Nash. On considère le cas de l'optimisation de forme vis-à-vis de deux disciplines $A$ et $B$ représentées par les critères $J_{A}$ et $J_{B}$, fonctions supposées régulières du vecteur $Y \in \mathbb{R}^{N}$ des variables de conception. Pour cela, on partage le vecteur $Y$ en deux sous-vecteurs $Y_{A}$ et $Y_{B}$,

$$
Y=\left(Y_{A}, Y_{B}\right)
$$

On utilise ces sous-vecteurs comme stratégies de deux joueurs virtuels qui optimisent respectivement $J_{A}$ et $J_{B}$ en tenant compte de la stratégie de l'autre jusqu'à ce qu'un équilibre soit atteint. Dans ce cas, on dit que $\bar{Y}=\left(\bar{Y}_{A}, \bar{Y}_{B}\right)$ réalise un équilibre de Nash ssi :

$$
\bar{Y}_{A}=\operatorname{Argmin}_{Y_{A}} J_{A}\left(Y_{A}, \bar{Y}_{B}\right) \text {, et } \bar{Y}_{B}=\operatorname{Argmin}_{Y_{B}} J_{B}\left(\bar{Y}_{A}, Y_{B}\right)
$$

En pratique, cet équilibre peut être atteint par un algorithme itératif dans lequel chaque joueur effectue, en parallèle aux autres, un certain nombre d'itérations d'optimisation de son critère propre avant d'échanger son meilleur itéré avec les autres, et ceci jusqu'à convergence. Ainsi, chaque 
discipline utilise sa propre méthode d'analyse adaptée à son propre jeu d'EDP, et son propre optimiseur, et n'interagit avec les autres que par l'échange de variables communes de conception. On pourra trouver des illustrations de telles optimisations multicritères en aérodynamique dans [5] et [6], et en électromagnétisme dans [7].

Comme on l'a mis en évidence dans [6] le choix qui est fait a priori du partage des variables, désigné ici partage de territoire, seul détermine la pertinence du compromis atteint à l'équilibre. De plus, des choix non physiques ne permettent pas d'atteindre cet équilibre. Par ailleurs, dans sa thèse [8], B. Abou El Majd a montré dans le cas d'un couplage aéro-structural, que l'aérodynamique est une discipline fragile, car sans précaution particulière, on peut atteindre un équilibre de Nash n'ayant aucune pertinence aérodynamique car le couplage induit des variations de forme non admissibles. Pour cette raison, on a bâti une stratégie particulière que l'on expose dans la section suivante, avant de généraliser notre approche.

\section{Cas d'une discipline fragile : l'aérodynamique}

Au plan théorique, on a proposé dans [9] une méthodologie pour le traitement numérique d'un problème d'optimisation concourante dans lequel deux critères sont à considérer, l'un, $J_{A}$, étant plus critique que le second, $J_{B}$. A convergence de la minimisation paramétrique, éventuellement sous contraintes, de la seule fonctionnelle principale, $J_{A}$, des approximations du gradient et de la matrice hessienne, ainsi que des $K$ gradients de contraintes actives sont par hypothèse disponibles ou calculées en utilisant des métamodèles. Alors, on partage l'espace paramétrique entier (un sousensemble de $\mathbb{R}^{N}$ ) en deux sous-espaces supplémentaires sur la base d'un critère lié à la seconde variation. La construction est telle qu'à partir du point initial de convergence de la minimisation de la fonctionnelle principale en dimension complète, des perturbations infinitésimales des paramètres dans le second sous-espace, dont la dimension $p \leq N-K$ peut être spécifiée, causent potentiellement la moindre dégradation de la valeur de la fonctionnelle principale. Ce sous-espace est choisi comme support de la stratégie de paramétrisation de la seconde fonctionnelle, $J_{B}$, dans une optimisation concourante mise en oeuvre par un algorithme simulant un jeu de Nash [10] entre des joueurs associés aux deux fonctionnelles respectivement. On a prouvé un deuxième résultat selon lequel le point optimum original du problème principal en dimension complète est Pareto-optimal vis-à-vis d'un problème trivial d'optimisation concourante. Ce dernier résultat nous a permis de définir un continuum de points d'équilibre de Nash ayant pour origine le point optimum initial, donnant ainsi au concepteur la possibilité de choisir un point opérationnel. Tertio, on a démontré que l'optimum initial monocritère était robuste.

Ces propriétés générales ont d'abord été mises en évidence dans le cas d'un problème simple de minimisation de formes quadratiques en dimension finie soumis à une contrainte d'égalité linéaire ou non-linéaire [9].

La méthodologie est ici illustrée en traitant un exercice académique mais difficile d'optimisation géométrique de la voilure d'un avion d'affaires vis-à-vis de critères aérodynamique et structural. En aérodynamique, on simule l'écoulement compressible extérieur par résolution en volumesfinis des équations d'Euler en trois dimensions d'espace, et on minimise le coefficient de traînée d'onde sous contrainte de portance. En calcul des structures, on traite la voilure comme une coque mince régie par les équations de l'élasticité linéaire (code ASTER de l'EDF), et on cherche à améliorer un critère intégral de contrainte. B. Abou El Majd [8] a testé divers jeux de Nash entre les deux critères, chaque variante étant caractérisée par un choix spécifique du partage des variables. Parmi les nombreux algorithmes testés, celui s'appuyant sur le partage issu de la diagonalisation du hessien restreint s'est clairement révélé le meilleur à la fois en stabilité de l'équilibre, et en performance du résultat atteint. A terme, le critère structural a été réduit de $8 \%$, au prix d'une augmentation de seulement $3 \%$ du critère aérodynamique, préservant ainsi à peu près l'écoulement préalablement optimisé vis-à-vis du seul critère aérodynamique (faible augmentation de l'intensité 
du choc, en rouge à la Figure 1).

a) Convergence des critères

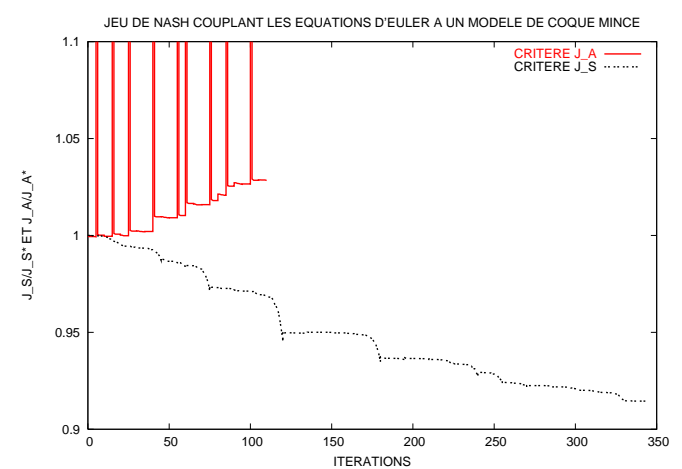

b) Optimum aérodynamique initial

c) Equilibre de Nash aéro-structural
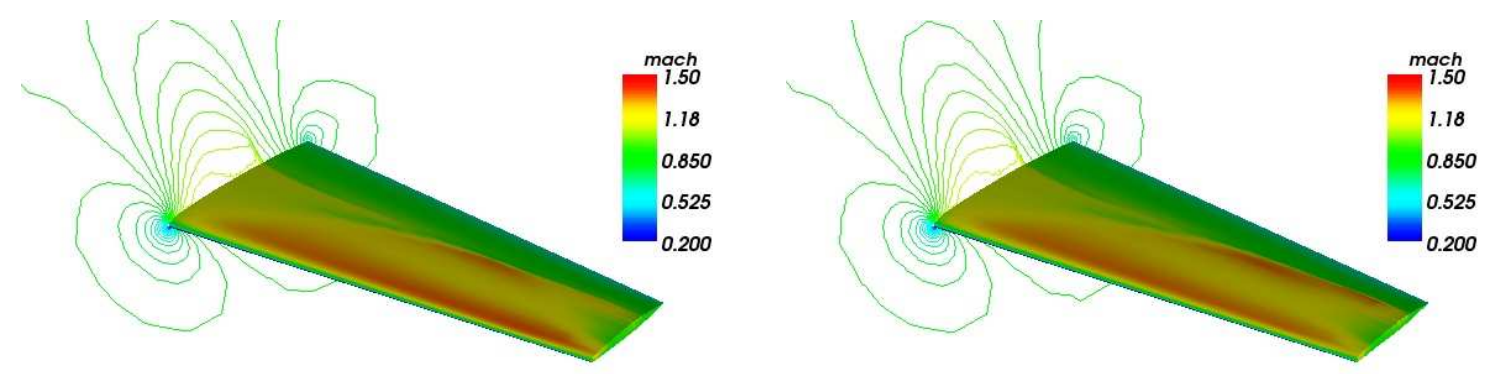

Figure 1 - Optimisation aéro-structurale d'une voilure; partage des variables par décomposition orthogonale : a) historique de convergence des critères (après 50 couplages à partir de l'optimum aérodynamique initial); champ de nombre de Mach correspondant à : b) l'optimum aérodynamique initial, et c) l'équilibre de Nash associé au partage des variables par décomposition orthogonale.

\section{Généralisation : stratégie d'optimisation à deux disciplines}

La stratégie de partage de territoire de la section précédente, valable lorsque l'une des disciplines est prépondérante ou fragile, et initialement optimisée seule, est ici généralisée au cas où le point de départ $Y^{0}$ est quelconque. Pour simplifier, on considère ici le cas d'optimisation sans contrainte. A nouveau, on suppose que gradients et hessiens sont disponibles quitte à construire des métamodèles pour les approcher. A fin de normalisation et d'amélioration du conditionnement, on peut leur associer des normes :

$$
\left\|H_{A}^{0}\right\|=\sqrt{\operatorname{trace}\left[\left(H_{A}^{0}\right)^{2}\right]} \quad\left\|H_{B}^{0}\right\|=\sqrt{\operatorname{trace}\left[\left(H_{B}^{0}\right)^{2}\right]}
$$

où l'indice supérieur ${ }^{0}$ indique une évaluation en $Y=Y^{0}$. Soit alors les nouveaux critères :

$$
\begin{aligned}
& \tilde{\tilde{J}_{A}}=\exp \left(\alpha \frac{\left\|H_{A}^{0}\right\|}{\left\|\nabla J_{A}^{0}\right\|^{2}}\left(J_{A}-J_{A}^{0}\right)\right)+\varepsilon_{0}\left[1-\chi_{\mathcal{B}_{R}}(Y)\right]\left(\frac{\left\|Y-Y^{0}\right\|}{R}-1\right)^{q+1} \\
& \tilde{\tilde{J}}_{B}=\exp \left(\beta \frac{\left\|H_{B}^{0}\right\|}{\left\|\nabla J_{B}^{0}\right\|^{2}}\left(J_{B}-J_{B}^{0}\right)\right)+\varepsilon_{0}\left[1-\chi_{\mathcal{B}_{R}}(Y)\right]\left(\frac{\left\|Y-Y^{0}\right\|}{R}-1\right)^{q+1}
\end{aligned}
$$


où $R$ est une échelle de $\mathbb{R}^{N}$ et $\mathcal{B}_{R}=\mathcal{B}\left(Y^{0}, R\right)$ est la boule de travail autour de $Y^{0}, \chi_{\mathcal{B}_{R}}(Y)$ sa fonction caractéristique, $\varepsilon_{0}$ un nombre positif petit, $q$ un entier tel que $J_{A}(Y)$ et $J_{B}(Y)$ sont au moins de classe $C^{q}(q \geq 2)$, et $\alpha, \beta$ et $\gamma$ des nombres positifs sans dimension tels que :

$$
\alpha \frac{\left\|H_{A}^{0}\right\|}{\left\|\nabla J_{A}^{0}\right\|}=\beta \frac{\left\|H_{B}^{0}\right\|}{\left\|\nabla J_{B}^{0}\right\|}=\frac{\gamma}{R}
$$

Alors, les nouveaux critères ont les propriétés suivantes :

- $\tilde{\tilde{J}}$ est sans dimension, strictement positif; sa variation dans la boule de travail est dans le même sens que celle de $J ; \tilde{\tilde{J}}$ est aussi de classe $C^{q}$;

- $\tilde{\tilde{J}}\left(Y^{0}\right)=1$ et $\lim _{\|Y\| \rightarrow \infty} \tilde{\tilde{J}}=\infty$, et pour un choix approprié des constantes $\alpha, \beta$ and $\gamma$ on a : $\left\|\nabla \tilde{\tilde{J}}_{A}^{0}\right\|=\left\|\nabla \tilde{\tilde{J}}_{B}^{0}\right\| \sim 1$

- Si $\bar{Y} \in \mathcal{B}_{R}$ réalise un équilibre de Nash entre les critères $J_{A}$ et $J_{B}$, c'est aussi le cas entre les critères $\phi\left(J_{A}\right)$ et $\psi\left(J_{B}\right)\left(\phi, \psi\right.$ monotones-croissantes), et en particulier entre $\tilde{\tilde{J}}_{A}$ et $\tilde{\tilde{J}}_{B}$.

Désormais, on remplace les critères initiaux par les nouveaux, et on omet la notation $\tilde{z}$.

Notre algorithme s'appuie sur un principe d'évidence : tout point qui n'est pas Pareto-optimal est améliorable vis-à-vis des deux critères simultanément. Cette observation nous conduit à proposer la définition suivante :

Définition [Pareto-stationnarité] : Soit deux critères $J_{A}$ et $J_{B}$ fonctions régulières (disons $C^{2}$ ) $d u$ vecteur $Y \in \mathbb{R}^{N}$. On dit que le point $Y^{0}$ est Pareto-stationnaire vis-à-vis des critères $\left(J_{A}, J_{B}\right)$ ssi il existe une combinaison convexe $\alpha J_{A}+(1-\alpha) J_{B}(0 \leq \alpha \leq 1)$ stationnaire en $Y^{0}$.

On peut décomposer les points Pareto-stationnaires en trois classes suivant le nombre de gradients nuls :

1. Type a : $\nabla J_{A}^{0}=\nabla J_{B}^{0}=0$

2. Type $\mathrm{b}: \nabla J_{A}^{0}=0$ et $\nabla J_{B}^{0} \neq 0$ (ou vice versa)

3. Type c : $\nabla J_{A}^{0}+\lambda \nabla J_{B}^{0}=0$ avec $\lambda=\frac{1-\alpha}{\alpha}>0$, ce qui est la condition de stationnarité satisfaite par les points Pareto-optimaux. (Stationnarité du lagrangien : $\mathcal{L}:=J_{A}+\lambda\left(J_{B}-C\right)$.)

Lorsque les critères sont localement convexes, la stratégie à adopter dans le cas où $Y^{0}$ est Pareto-stationnaire est assez évidente suivant le type :

1. Pareto-stationnarité de type a : $\nabla J_{A}^{0}=\nabla J_{B}^{0}=0$. Dans ce cas exceptionnel où les deux critères sont simultanément (localement) minimaux, on interrompt l'optimisation.

2. Pareto-stationnarité de type $\mathrm{b}:$ disons $\nabla J_{A}^{0}=0$ et $\nabla J_{B}^{0} \neq 0$. Alors $J_{A}$ est minimum, et $J_{B}$ perfectible : on bien on s'arrête, ou bien on est ramené au cas de la section précédente, pour lequel un jeu de Nash avec un certain partage de territoire fait sens.

3. Pareto-stationnarité de type c : $\nabla J_{A}^{0}+\lambda \nabla J_{B}^{0}=0(\lambda>0)$. Dans ce cas, le point est Paretooptimal et l'optimisation est interrompue.

Ces résultats s'aménagent au cas de critères non convexes par examen des systèmes propres des matrices hessiennes, $H_{A}^{0}$ et $H_{B}^{0}$. Nous ne pouvons donner le détail ici. Néanmoins, notons que le cas d'un point $Y^{0}$ Pareto-stationnaire de type $\mathrm{c}$ se ramène à celui de Pareto-stationnarité de type a dans l'hyperplan orthogonal à la direction commune $u_{A B}$ des gradients, ce qui conduit à examiner les systèmes propres des matrices hessiennes réduites :

$$
H_{A}^{\prime 0}=P_{A B} H_{A}^{0} P_{A B} \quad{H_{B}^{\prime}}^{0}=P_{A B} H_{B}^{0} P_{A B}
$$

où $P_{A B}$ est la matrice de projection $P_{A B}=I-\left[u_{A B}\right]\left[u_{A B}\right]^{t}$.

Enfin, dans le cas banal inverse où $Y^{0}$ n'est pas Pareto-stationnaire, on pose :

$$
u=\nabla J_{A}^{0} \quad v=\nabla J_{B}^{0} \quad w=\frac{\|v\| u+\|u\| v}{\|v\|+\|u\|}
$$


Il est alors facile de montrer que

$$
w . u>0 \quad w . v>0
$$

Autrement dit, la direction $-w$ est une direction de descente pour les deux critères simultanément. On fait donc un pas dans la direction de $-w$, on recalcule les gradients, et si le point atteint n'est pas Pareto-stationnaire, on recommence, et ainsi de suite.

Deux éventualités sont possibles. Ou bien on atteint un point Pareto-stationnaire en un nombre fini de pas, et on a déjà défini notre stratégie dans ce cas. Ou bien la suite est infinie, mais alors :

- Les suites infinies de valeurs des critères $\left\{J_{A i}\right\}$ and $\left\{J_{B i}\right\}$ qui sont strictement monotonesdécroissantes et positives, sont alors également bornées.

- Puisque les critères sont infinis à l'infini, la suite des points de conception $\left\{Y^{i}\right\}$ est ellemême bornée.

- Il existe donc une sous-suite infinie convergente. Soit $Y^{*}$ la limite.

On fait la conjecture que le point $Y^{*}$ est lui-même Pareto-stationnaire, ce qui nous ramène au cas précédent. (Sinon on réinitialise $Y^{0}$ à $Y^{*}$.)

En conclusion, dans le cas général, on propose de conduire d'abord un plan d'expérience à partir d'un jeu convenable de valeurs du point de départ $Y^{0}$. Puis, à partir de chaque point, conduire une phase d"'optimisation coopérative", à chaque itération de laquelle les deux critères diminuent simultanément, et jusqu'à atteindre un point Pareto-stationnaire. Alors, s'arrêter, ou conduire une phase d"'optimisation concurrentielle" par un jeu de Nash associé à un partage de territoire basé sur l'analyse des systèmes propres des matrices hessiennes locales.

\section{Références}

[1] T.W. Sederberg and S.R. Parry. Free-From Deformation of Solid Geometric Models. Computer Graphics, 20(4) :151-160, 1986. See also : Computer Aided Geometric Design, Sederberg, T.W., http ://tom.cs.byu.edu/ tom/ (Item : Courses).

[2] B. Abou El Majd, J.-A. Désidéri, and R. R. Duvigneau. Multilevel strategies for parametric shape optimization in aerodynamics. European Journal of Computational Mechanics, 17(12), 2008

[3] N. Srinivas and K. Deb. Multi-objective function optimization using non-dominated sorting genetic algorithms. Evolutionary Computation, 2(3):221 248, 1995.

[4] D.E. Goldberg. Genetic Algorithms in Search, Optimization and Machine Learning. Addison Wesley Company Inc., 1989.

[5] J.F. Wang. Optimisation Distribuée Multicritère par Algorithmes Génétiques et Théorie des Jeux \& Application à la Simulation Numérique de Problèmes d'Hypersustentation en Aérodynamique. PhD thesis, University of Paris 6, 2001. Spéc. : Math. App.

[6] Z. Tang, J.-A. Désidéri, and J. Périaux. Multi-Criterion Aerodynamic Shape-Design Optimization and Inverse Problems Using Control Theory and Nash Games. Journal of Optimization Theory and Applications, 2007.

[7] M. Sefrioui. Algorithmes Evolutionaires pour le Calcul Scientifique, Application à l' Electromagnétisme et à la Mécanique des Fluides Numérique. PhD thesis, Paris 6, Paris, France, 1998.

[8] B. Abou El Majd. Algorithmes hiérarchiques et stratégies de jeux pour l'optimisation multidisciplinaire - Application à l'optimisation de la voilure d'un avion d'affaires. $\mathrm{PhD}$ thesis, Université de Nice-Sophia Antipolis, 2007.

[9] J.-A. Désidéri. Split of Territories in Concurrent Optimization. Research Report 6108, INRIA, January 2007. URL : https ://hal.inria.fr/inria-00127194.

[10] J. F. Nash. Non-Cooperative Games. Annals of Mathematics, 54(2) :286-295, 1951. 